\title{
GEOPHYSICS
}

\section{Passive seismic event estimation using multi-scattering waveform inversion}

\begin{tabular}{|r|l|}
\hline Journal: & Geophysics \\
\hline Manuscript ID & GEO-2018-0358.R4 \\
\hline Manuscript Type: & Technical Paper \\
\hline Keywords: & $\begin{array}{l}\text { microseismic, passive seismic, full-waveform inversion, frequency- } \\
\text { domain }\end{array}$ \\
\hline Area of Expertise: & Passive Seismic and Microseismic Methods, Seismic Inversion \\
\hline \multicolumn{2}{|l}{} \\
\hline
\end{tabular}

\section{SCHOLARONE \\ Manuscripts}


Passive seismic event estimation using multi-scattering waveform inversion

\author{
Chao Song, Zedong Wu, Tariq Alkhalifah \\ King Abdullah University of Science and Technology (KAUST), \\ Physical Sciences and Engineering Division, \\ Thuwal, Saudi Arabia, 23955
}

(December 31, 2018)

Running head: Passive seismic event estimation

\begin{abstract}
Passive seismic monitoring has become an effective method to understand underground processes. Time-reversal-based methods are often used to locate passive seismic events directly. However, these kinds of methods are strongly dependent on the accuracy of the velocity model. Full waveform inversion (FWI) has been employed on passive seismic data to invert the velocity model and source image, simultaneously. However, waveform inversion of passive seismic data utilizes mainly the transmission energy, which results in poor illumination and low resolution. We develop a waveform inversion using multi-scattered energy for passive seismic to extract more information from the data than conventional FWI. Using transmission wavepath information from single- and double-scattering, computed from a predicted scatterer field acting as secondary sources, the proposed method provides better illumination of the velocity model than conventional FWI. Using a new objective function, we optimize the source image and velocity model including multi-scattered energy, simultaneously. As we conduct our method in the frequency domain with a complex source function including both spatial and wavelet information, we mitigate the uncertainties of the source wavelet and source origin time. Inversion results from the Marmousi model show that by taking advantage of multi-scattered energy and starting from a reasonably acceptable frequency (single source at $3 \mathrm{~Hz}$ and multiple sources at $5 \mathrm{~Hz}$ ), the proposed method yields better inverted velocity models and source images compared with the conventional FWI.
\end{abstract}




\section{INTRODUCTION}

Passive seismic or induced seismicity refers to minor earthquakes caused by human activities, such as mining, hydrocarbon extraction and hydraulic fracturing. Passive seismic monitoring provides important information of subsurface processes. The most popular applications include fracture mapping, reservoir fluid migrations and production monitoring (Warpinski, 2009). All this information is crucial for engineers to optimize injection strategies.

Over the years, many methods to locate passive seismic events have been suggested. Raybased methods utilize traveltime picking of either P-wave or S-wave arrivals (Waldhauser and Ellsworth, 2000; Eisner et al., 2009; Diekmann et al., 2018). Though efficient and reasonably reliable, this category of methods tend to fail when the signal-to-noise ratio (SNR) is low and traveltime picking is not accurate. Time-reversal (Gajewski and Tessmer, 2005; Artman et al., 2010; Sava, 2011) and Gaussian-beam migration (Rentsch et al., 2004) based methods are also widely used. These methods utilize the wave equation to backward (reverse time) reconstruct the wavefield. Using an appropriate imaging condition, passive seismic source locations can be determined (Rocha et al., 2018). This category of methods require accurate velocity models. The uncertainties of the source wavelet and source origin time often make the wavefield reconstruction result unreliable (Song et al., 2017). Unlike active seismic experiments, such information is usually unavailable in the passive seismic case. Witten and Shragge (2017) proposed to separate P- and S-wave modes and get the source image calculated by crosscorrelating the adjoint P- and S-wavefields. Recently, full waveform inversion (FWI) has been utilized to estimate the passive seismic source location. Kamei and Lumley (2014) updated the velocity model via an FWI algorithm, but they assumed the background velocity is known and just inverted the velocity perturbation in the fluid injection area. Kaderli et al. (2015) proposed to separate the wave equation source term into spatial and temporal components. They used FWI to invert for the source components. Thus, they assume the true velocity model is known. However, an inaccurate velocity model of the subsurface usually leads to inaccurate passive seismic events determination. In order to solve this problem, Wang and Alkhalifah (2016) developed a source-independent full waveform inversion method to invert for the velocity model, as well as the passive seismic 
source image. By convolving reference traces with the observed and modeled data (Choi and Alkhalifah, 2011), the effect of an unknown source origin time can be mitigated. By inverting the source wavelet and the velocity model, the source location can be accurately determined. Wu and Alkhalifah (2017b) proposed a source-extension approach to determine the source location while inverting for the velocity and source image, simultaneously in the frequency domain. Some researchers also developed feasible strategies to incorporate timereversal source imaging based methods, as well as FWI based methods to invert for a good velocity model and source image inversion results (Sun et al., 2016).

Most of the work mentioned above is based on the conventional FWI with the leastsquares misfit between observed data with calculated data (Tarantola, 1984). In the conventional FWI, the low-wavenumber update in the velocity model comes primarily from transmitted waves (Virieux and Operto, 2009). Using scattered energy, FWI can provide high-resolution velocity models for the active seismic case. However, in passive seismic cases, because of the limited number of sources at depth, the inversion is dominated by transmitted wave energy, and thus, result in low-resolution velocity models with limited illumination. Low-wavenumber components can only be updated in the shallow part of the velocity model along the transmission wavepath. By extracting transmitted wave information from the single- or double-scattered wavefield computed from a predicted scattered field acting as secondary sources, we provide better illumination to the model (Alkhalifah and $\mathrm{Wu}, 2016 \mathrm{a})$. A new objective function was proposed to provide multi-scattering inversion (Alkhalifah and $\mathrm{Wu}, 2014,2016 \mathrm{~b}$ ). This new objective function provides the possibility to generate the velocity gradient with multi-scattered energy. In order to improve the computational efficiency, Wu and Alkhalifah (2015) proposed to update the background velocity and velocity perturbation (model image) simultaneously using the new objective function.

In this article, we use the wave equation in an optimization problem to invert the source image and velocity model including the multi-scattered energy simultaneously in the frequency domain. Unlike the conventional time-domain wave equation used in passive seismic source image inversion (Wang and Alkhalifah, 2016), which separates the source term into the spatial and wavelet components, we incorporate the spatial and wavelet parts 
into one source function made of complex values. By doing so, the uncertainties of the source wavelet and source origin time of passive seismic source can be mitigated. To further improve the conventional FWI used in the passive seismic data (Wu and Alkhalifah, 2017b), we use a new objective function to measure the misfit, which allows to get the velocity gradient including multi-scattered energy. In order to enforce a focused source image, we add a penalty term to the misfit function. This penalty function can also be used as an objective function to optimized the source image and velocity model simultaneously which is discussed in our following paper (Song et al., 2019). To mitigate the effect of the large model space given by the source image and velocity models, we incorporate a total variation (TV) regularization to reduce the inversion artifacts related to source encoding. Finally, we test the proposed method on the Marmousi velocity model using the data without low frequency information, starting with a linearly increasing with depth velocity model.

\section{THEORY}

The success of the conventional FWI in the active seismic case relies on obtaining smooth updates at the early stage using mainly transmission or diving waves. However, due to acquisition geometry limits in reality, diving waves cannot always be recorded in the dataset. In order to achieve this, reflection waveform inversion (RWI) was developed to generate the first-order scattering and provide smooth update along the reflection raypath (Xu et al., 2012; Zhou et al., 2012; Yao and Wu, 2017). In passive seismic case, due to the limited source number and poor illumination, single-, double-, or multi-scattered components in the data are usually ignored, and this contributes to the non-linear data mismatch. The observed data consists of the data from source function, as well as scattered energy from the model image. We propose to combine all this information into a single objective function. Using the $l_{2}$ norm misfit between observed data $d_{o}$, and the predicted data, the new objective function of summing over all the passive sources and the selected frequency band is stated as (Alkhalifah, 2017):

$$
E\left(m, \delta m, f, \mathbf{x}_{\mathbf{s}}\right)=\frac{1}{2} \sum_{\omega} \sum_{i}\left|d_{o_{i}}-d_{f_{i}}(m, f, \omega)-d_{m 1_{i}}(m, \delta m, \omega)-d_{m 2_{i}}(m, \delta m, \omega)\right|^{2}
$$




$$
+\alpha J_{f}\left(\mathbf{x}_{\mathbf{s}}, f\right)+\beta J_{T V}(m)
$$

where $i$ is the passive seismic source index, $\omega$ is the angular frequency, $m(\mathbf{x})=\left(1 / v^{2}\right)$ represents the velocity model information, $d_{f}(m, f, \omega)=u_{s}\left(\mathbf{x}=\mathbf{x}_{\mathbf{r}}\right), d_{m 1}(m, \delta m, \omega)=$ $\delta u_{s}\left(\mathbf{x}=\mathbf{x}_{\mathbf{r}}\right)$ and $d_{m 2}(m, \delta m, \omega)=\delta^{2} u_{s}\left(\mathbf{x}=\mathbf{x}_{\mathbf{r}}\right)$ are the predicted data from the source function $f(\mathbf{x}, \omega)$, single- and double-scattered model image $\delta m(\mathbf{x})$ at the receivers locations $\mathbf{x}_{\mathbf{r}}, \mathbf{x}=[x, y, z]$ denotes spatial dimensions. In order to focus the source energy, we add a source focusing term $J_{f}=\frac{\sum_{\mathbf{x}}\left|\mathbf{x}-\mathbf{x}_{\mathbf{s}}\right|^{2} \sum_{\omega}|f(\mathbf{x}, \omega)|^{2}}{\sum_{\mathbf{x}} \sum_{\omega}|f(\mathbf{x}, \omega)|^{2}}$ to the objective function, and $\mathbf{x}_{\mathbf{s}}$ represents the estimated source location coordinate. The basic idea is to penalize non-physical source energy away from $\mathbf{x}=\mathbf{x}_{\mathbf{s}}$ in the source image. This function is originally proposed to use as an extended source function to replace the point source which aims at solving the cycle-skipping problem in FWI (Huang and Symes, 2016). In order to estimate the passive source locations, Wu and Alkhalifah (2017b) described the source term as $f(\omega) w(\mathbf{x})$, which means spatial and wavelet components multiplication. If we want to invert for the spatial component $w(\mathbf{x})$, wavelet information $f(\omega)$ has to be estimated first. The source function $f(\mathbf{x}, \omega)$ consists of both wavelet and spatial information, and the amplitude of $f(\mathbf{x}, \omega)$ represents the spatial feature of the source function, which is referenced as the source image $|f(\mathbf{x}, \omega)|$. As we implement our method in the frequency domain, so the uncertainty of the passive source origin time in the time domain FWI is no longer a problem. In order to make the velocity update smooth, while still preserving the edges of the velocity perturbation, we use the TV regularization as a penalty term $J_{T V}=\sum_{\mathbf{x}} \sqrt{m_{\mathbf{x}}^{2}+\varepsilon}$, where $\varepsilon$ is a small number to make $J_{T V}$ differentiable at the origin, and $m_{\mathbf{x}}=\frac{\partial m}{\partial \mathbf{x}}$ (Vogel, 2002; Zhdanov, 2002). The $\alpha$ and $\beta$ are weights of the source penalty term and TV regularization term, respectively. Source wavefield $u_{s}$, single-scattered wavefield $\delta u_{s}$ and double-scattered wavefield $\delta^{2} u_{s}$ is calculated using (Zhang and Duan, 2012)

$$
\begin{aligned}
& L(m, \omega) u_{s}=f(\mathbf{x}, \omega), \\
& L(m, \omega) \delta u_{s}=-\omega^{2} \delta m(\mathbf{x}) u_{s}(\mathbf{x}, \omega), \\
& L(m, \omega) \delta^{2} u_{s}=-\omega^{2} \delta m(\mathbf{x}) \delta u_{s}(\mathbf{x}, \omega),
\end{aligned}
$$


where $L(m, \omega)=\nabla^{2}+\omega^{2} m(\mathbf{x})$ is the Helmholtz wave equation operator. As we use a source-space function to calculate the source wavefield, the assumption of a point source is relaxed to extend the velocity search space. The extension of the source image can help us fit the data when the velocity is inaccurate. With the help of the new objective function, we are capable of optimizing the velocity $m(\mathbf{x})$ with multi-scattered energy. We first ignore the influence of double-scattered energy, which indicates $d_{m 2}(m, \delta m, \omega)=0$. We need to obtain a model image $\delta m(\mathbf{x})$ to calculate the single-scattered data $d_{m 1}(m, \delta m, \omega)$. The model image gradient $\nabla_{\delta m} E$, via adjoint-state method (Fernandez-Berdaguer, 1998; Plessix, 2006), is given by

$$
\nabla_{\delta m} E=\frac{\partial E}{\partial \delta m}=-\Re\left\{\sum_{\omega} \omega^{2} u_{s}(\mathbf{x}, \omega) u_{r}(\mathbf{x}, \omega)\right\}
$$

where $\Re$ stands for the real part of a complex number, $u_{r}(\mathbf{x}, \omega)$ is the adjoint wavefield. The model image is calculated by stacking over the full spectrum. The adjoint wavefield is evaluated by solving

$$
L^{t}(m, \omega) u_{r_{i}(\omega)}=\sum_{j} \delta\left(\mathbf{x}-\mathbf{x}_{\mathbf{r}_{\mathbf{j}}}\right)\left(d_{o_{i j}}-d_{f_{i j}}(m, f, \omega)-d_{m 1_{i j}}(m, \delta m, \omega)\right)^{*}
$$

where $\mathbf{x}_{\mathbf{r}_{\mathbf{j}}}$ is the receiver position, $j$ is the receiver index, $L^{t}(m, \omega)$ is the adjoint operator, * represents the complex conjugate. The velocity model gradient of this objective function including single-scattered energy is given by

$$
\frac{\partial E}{\partial m}=-\Re\left\{\omega^{2}\left(u_{s} u_{r}+u_{s} \delta u_{r}+u_{r} \delta u_{s}\right)\right\}-\beta \nabla \cdot \frac{\nabla m}{J_{T V}} .
$$

In equation $5, R_{F W I}=-\Re\left(\omega^{2} u_{s} u_{r}\right)$ corresponds to the conventional FWI velocity model gradient with no scattered energy, $R_{\text {single }}=-\Re\left\{\omega^{2}\left(u_{s} \delta u_{r}+u_{r} \delta u_{s}\right)\right\}$ corresponds to the velocity model gradient of the single-scattered energy, and $\beta \nabla \cdot \frac{\nabla m}{J_{T V}}$ corresponds to the velocity model gradient of the TV regularization term. The single-scattered adjoint wavefield $\delta u_{r}$ is calculated using

$$
L^{t}(m, \omega) \delta u_{r}=-\omega^{2} \delta m(\mathbf{x}) u_{r}(\mathbf{x})
$$


If we want to calculate the source function gradient, we need to evaluate $\mathbf{x}_{\mathbf{s}}$ for $f(\mathbf{x}, \omega)$. Analytically, it is given by

$$
\mathbf{x}_{\mathbf{s}}=\frac{\sum_{\mathbf{x}} \sum_{\omega}|f(\mathbf{x}, \omega)|^{2} \mathbf{x}}{\sum_{\mathbf{x}} \sum_{\omega}|f(\mathbf{x}, \omega)|^{2}}
$$

The gradient of source function $f(\mathbf{x}, \omega)$ is then given by

$$
\begin{aligned}
\frac{\partial E}{\partial f}= & \sum_{\omega}\left(u_{r}(\mathbf{x}, \omega)\right)+2 \alpha \sum_{\omega}|f(\mathbf{x}, \omega)| \frac{\sum_{\mathbf{x}}\left|\mathbf{x}-\mathbf{x}_{\mathbf{s}}\right|^{2} \sum_{\omega}|f(\mathbf{x}, \omega)|^{2}}{\sum_{\mathbf{x}} \sum_{\omega}|f(\mathbf{x}, \omega)|^{2}}- \\
& \left.\frac{\sum_{\mathbf{x}}\left|\mathbf{x}-\mathbf{x}_{\mathbf{s}}\right|^{2} \sum_{\omega}|f(\mathbf{x}, \omega)|^{2}}{\left(\sum_{\mathbf{x}} \sum_{\omega}|f(\mathbf{x}, \omega)|^{2}\right)^{2}}\right) .
\end{aligned}
$$

We further consider the influence from both single- and double-scattered energy. We derive the gradient of squared slowness model $m(\mathbf{x})$ and model image $\delta m(\mathbf{x})$ from doublescattered wavefield using the extended Lagrangian formulation, which is described in detail in Alkhalifah and $\mathrm{Wu}(2016 \mathrm{a}) . d_{m 2}(m, \delta m, \omega)$ is the double-scattered data extracted from the double-scattered wavefield $\delta^{2} u_{s}$, which can be calculated using equation 2 . The model image $\delta m(\mathbf{x})$ gradient including both single- and double-scattered energy is:

$$
\frac{\partial E}{\partial \delta m}=-\Re\left\{\sum_{\omega} \omega^{2}\left(u_{s} u_{r}+u_{s} \delta u_{r}+\delta u_{s} u_{r}\right)\right\}
$$

The adjoint wavefields $u_{r}$ and $\delta u_{r}$ can be calculated by

$$
\begin{gathered}
L^{t}(m, \omega) u_{r_{i}(\omega)}=\sum_{j} \delta\left(\mathbf{x}-\mathbf{x}_{\mathbf{r}_{\mathbf{j}}}\right)\left(d_{o_{i j}}-d_{f_{i j}}(m, f, \omega)-d_{m 1_{i j}}(m, \delta m, \omega)-d_{m 2_{i j}}(m, \delta m, \omega)\right)^{*} . \\
L^{t}(m, \omega) \delta u_{r}=-\omega^{2} \delta m(\mathbf{x}) u_{r}(\mathbf{x}) .
\end{gathered}
$$

The gradient of the of the objective function with respect to squared slowness model including both single- and double-scattered energy can be derived as:

$$
\frac{\partial E}{\partial m}=R_{F W I}+R_{\text {single }}-\Re\left\{\sum_{\omega} \omega^{2}\left(u_{s} \delta^{2} u_{r}+\delta u_{s} \delta u_{r}+\delta^{2} u_{s} u_{r}\right)\right\} .
$$

The adjoint wavefield $u_{r}$ satisfies

$$
L^{t}(m, \omega) \delta^{2} u_{r}=-\omega^{2} \delta m(\mathbf{x}) \delta u_{r}(\mathbf{x})
$$




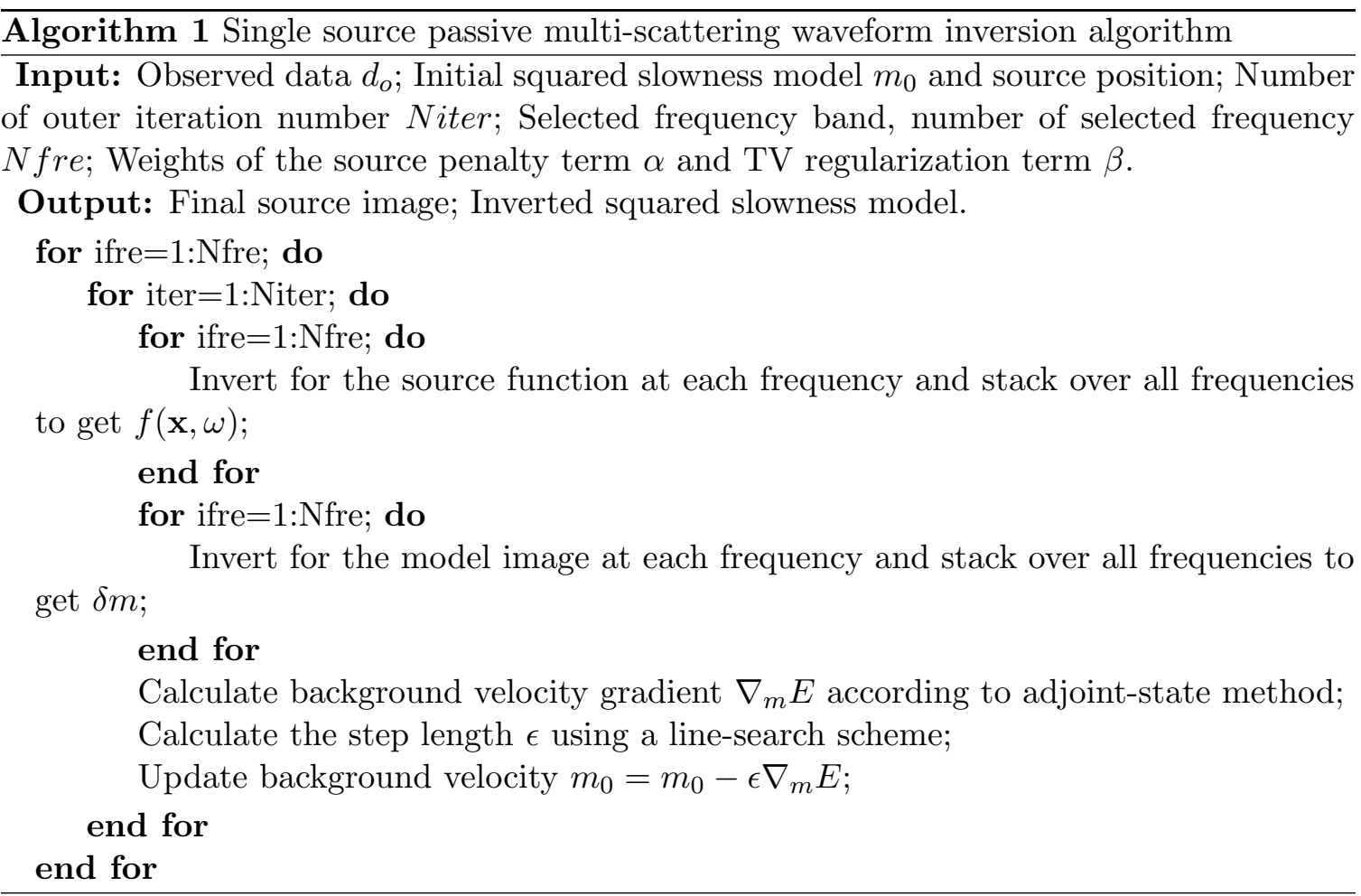

The source function gradient is still given by equation 9 when we also consider doublescattered energy, and the formulation to calculate $\mathbf{x}_{\mathbf{s}}$ also remains the same as equation 8 . To sum up, the workflow is shown as Algorithm 1.

\section{SENSITIVITY KERNELS}

In the early stage of the conventional FWI, low-wavenumber components in the velocity model are gradually recovered thanks to the transmission and diving waves. In this stage, scattered energy cannot be utilized. So here we call this early stage of the conventional FWI as zero-scattered FWI stage. In order to analyse the behaviour of the zero-scattered FWI and multi-scattered FWI velocity gradients in the passive seismic case, we need to evaluate their sensitivity kernels. Zero-scattered FWI and single-scattered sensitivity kernels are calculated as $R_{F W I}=-\Re\left(\omega^{2} u_{s} u_{r}\right)$ and $R_{\text {single }}=-\Re\left\{\omega^{2}\left(u_{s} \delta u_{r}+u_{r} \delta u_{s}\right)\right\}$ which are discussed in the theory part. Firstly, we use a simple two-layer model of size $120 \times 120$ samples. The horizontal and vertical grid intervals are $25 \mathrm{~m}$. The interface between the two layers is at $2.25 \mathrm{~km}$ depth. The source location is denoted as the $*$ symbol, and the 
receiver location is shown as the $\triangle$ symbol, in Figure 1 . In this paper, the shallow part indicates the area above the source location, and the deep part indicates the area beneath the source location. The sensitivity kernels of the zero-scattered and single-scattered FWI kernels for the horizontal layer model are shown in Figure 2a and 2b, respectively. In our proposed approach, we combine the zero-scattered and single-scattered kernels to cover a larger area with low-wavenumber velocity model update shown in Figure 3. By doing so, we can access more background velocity information which is often ignored in passive seismic data inversion, and even in global seismology (Bozdag et al., 2011), and this will lead to a better source image inversion result, as more energy will be focused back to the source location.

From the velocity sensitivity kernels analysis, we realize that the single-scattered energy provides smooth updates in the deeper part of the horizontal layer model. So what will the multi-scattered velocity gradient provide in the dipping layered model? The source and receiver locations are shown using the same symbols as in Figure 4. We also use the same background velocity model as previous example. The sensitivity kernels of the zeroscattered FWI and single-scattered FWI for the dipping layer model are shown in Figure 5. The combined FWI kernel with single-scattered energy for the dipping layer model is shown in Figure 6.

From the above example, we see that in the dipping model case, the FWI velocity gradient with single-scattered energy will provide better illumination in the shallow part of the velocity model. By comparison, the zero-scattered FWI velocity gradient with no scattered energy can only update the low-wavenumber components along the transmission wavepath. Both Figure 3 and 6 leads to the same conclusion that: taking advantage of multi-scattered energy leads to an improvement of the illumination of the velocity model. As a result, the velocity inversion is expected to converge faster than the zero-scattered FWI. 


\section{SOURCE IMAGE INVERSION}

There are two main steps in the proposed method. The first step is to update the velocity model, and the second step is to invert for the source image. After showing that the FWI velocity model gradient for multi-scattered energy provides better illumination, we want to find out if this approach can also generate better source image inversion results than the zero-scattered FWI. We use a homogeneous model with one strong anomaly. We place receivers at all four boundaries to have a perfect acquisition system. The size of the model is $400 \times 400$. The true source position is shown as the $*$ symbol in Figure 7 . The wavelet we use is a Ricker wavelet with a peak frequency of $6 \mathrm{~Hz}$. We apply the inversion with a frequency range of $5 \mathrm{~Hz}$ to $7 \mathrm{~Hz}$ with a frequency interval of $0.1 \mathrm{~Hz}$. We use the true velocity to invert the source image. Within the source focusing property term $J_{s}$, if the distance $\left|\mathbf{x}-\mathbf{x}_{\mathbf{s}}\right|^{2}$ is large, it will increase the singularity of the penalty function. In order to mitigate this, we smooth the penalty function toward the edges, as shown in Figure 8. First, we use the conventional FWI method to invert the source image with no multiscattered energy involved. After 20 inner iterations (no change in the background velocity), the inverted source image is shown in Figure 8a. Then, we use FWI with single-scattered energy to invert the source image and model image, simultaneously. When we invert the source image, the background velocity model remains the same. As the LU decomposition of the impedance matrix is the most time-consuming part, we just need to use the previously stored L and U matrices. So the iterations used to update the source image and velocity model are computationally inexpensive. After the same number of inner iterations, the inverted source image is shown in Figure 9b, and the model image is shown in Figure 10. From the source image inversion results, we see that the source energy is better concentrated using single-scattered FWI, and the output high-resolution source image can easily tell us the correct source location (as the black $*$ symbol denotes). That is because by obtaining the model image, we calculate the scattered wavefield and relocate the scattered energy back to the source location. 


\section{MARMOUSI MODEL}

We further apply the proposed method on part of the Marmousi model of size $240 \times 100$ samples, as shown in Figure 11. For the initial model we use a linearly increasing velocity with depth, shown in Figure 12a. The horizontal and vertical grid interval is $25 \mathrm{~m}$. In this case, we use a Ricker wavelet with the peak frequency being $6 \mathrm{~Hz}$. Since low frequencies are often contaminated by noise, we start the inversion here from $3 \mathrm{~Hz}$ up to $6 \mathrm{~Hz}$ with a frequency interval of $0.15 \mathrm{~Hz}$. To limit the leakage of energy from the source function and model image to the velocity model, we use an efficient scattering-angle enrichment filter initially on the velocity gradient to have only smooth updates (Wu and Alkhalifah, 2017a). All the grid points on the surface act as receivers. The true source coordinate location is $(4.625 \mathrm{~km}, 2.0 \mathrm{~km})$, and the initial source coordinate location for inversion is $(4.625 \mathrm{~km}, 1.5$ $\mathrm{km})$. The $\alpha$ and $\beta$ in the objective function we use is $1.0^{-10}$ and 0.1 , and we choose them based on tests conducted beforehand. The value of $\alpha$ is much smaller, and that is because the value of the source image is relatively small. For zero- and single-scattered FWI, we perform 80 outer loop iterations to update the velocity. But for double-scattered FWI, the convergence speed is significantly faster, so we just need to perform 20 outer loop iterations to complete the velocity inversion. In the inner loop, we use 20 computationally inexpensive iterations (since the wave equation operator is the same) to optimize the source function and the model image.

The final inverted velocity model obtained by zero-, single- and double-scattered FWI are shown in Figure 12b, 12c and 12d, respectively. The inverted velocity model from the zero-scattered FWI cannot capture the structure of true velocity model. By comparison, FWI with multi-scattered energy will generate better velocity inversion results with limited additional cost. The inversion results have more of the true model structure, in spite of the fact that this inversion corresponds to a single source. We see that FWI with single- and double-scatted energy can both reconstruct the velocity model in the deep part, and the double-scatted FWI can build better velocity models in the shallow part. These features are consistent with behaviour the sensitivity kernels discussed previously. 
The source image inverted using the initial velocity model after 20 inner loop within the first outer-loop iteration is shown in Figure 13a. Since the initial velocity model is very far from the true model, the source energy is evidently not focused. Alternatively, the inverted source images of the zero- and single-scattered FWI after 80 iterations are shown in Figure $13 \mathrm{~b}$ and $13 \mathrm{c}$, respectively. The inverted source image of the double-scattered FWI after 20 iterations is shown in Figure 13d. The $\triangle$ symbol represents the true location of the source, and the $*$ symbol represents the initial source position for the source image inversion. The inverted source images using zero-, single- and double-scattered inverted velocity models are well focused. However, when we take a second look at the source image in Figure 13b, we may find that the source energy in the inverted source image using zero-scattered FWI inverted velocity model is not focused at the true source position. By comparison, the source energy in the inverted source images from single- and double-scattered FWI inverted velocity models are focused at the true source location, as demonstrated in Figure 13c and $13 \mathrm{~d}$. In order to analyze the ability of the proposed method, we add Gaussian noise with a SNR of $1 \mathrm{~dB}$ to the synthetic data. With the same frequency band and inversion strategy, the inverted velocity and source image using the zero-scattered FWI are shown in Figures 14a and 14b. Meanwhile, the inverted velocity and source image using the double-scattered FWI are shown in Figures $14 \mathrm{c}$ and $14 \mathrm{~d}$. We see that with high levels of noise in the data, the zero-scattered FWI cannot image the main structures of the true model, and the source energy does not focus at the true source location. By comparison, the double scattered FWI still manages to produce a reasonable velocity model. Even though there are more artifacts in the inverted source image using the noisy data, and the source energy still focuses at the true source location, with only a slight shift.

The above shown examples are all based on a single source. We want to test if the proposed method is valid for multiple sources. We use the same true and initial velocity model and place three sources in the model with coordinates $(2.5 \mathrm{~km}, 2.0 \mathrm{~km}),(5.0 \mathrm{~km}, 1.75$ $\mathrm{km})$ and $(7.5 \mathrm{~km}, 1.5 \mathrm{~km})$. The common initial source position coordinate is $(4.625 \mathrm{~km}, 1.5$ $\mathrm{km})$. In this case, we start the passive multi-scattering FWI from higher frequency, specially from $5 \mathrm{~Hz}$ to $8 \mathrm{~Hz}$ with a frequency interval of $0.15 \mathrm{~Hz}$. After completing a simultaneous 
velocity model and source image inversion, the inverted velocity models using zero-, single-, and double-scattered energy are shown in Figure 15b, 15c and 15d, respectively. Zeroscattered FWI fails to build the main structure of true velocity model shown in Figure 15b. Even though we use the efficient scattering-angle enrichment filter to smooth the velocity gradient, there are still some discontinuous high-wavenumber components in the inverted velocity model. FWI using multi-scattered energy can almost recover a smooth version of the true velocity model.

The source image with three sources inverted from the initial velocity model after 20 inner loop of the first outer-loop iteration is shown in Figure 16a. With such an inaccurate velocity, the source energy cannot be well focused on the true source location. The inverted source images corresponding to final inverted velocity models from zero-, single- and doublescattered FWI are shown in Figure 16b, 16c and 16d, respectively. From Figure 16b, we see that only one source on the right is well captured and the energy of the middle source is relatively weak. In addition, the event on the left bottom can barely be located. Let us look at the inverted source images from single- and double-scattered FWI inverted velocity models demonstrated in Figure $16 \mathrm{c}$ and 16d, respectively. The two events in the middle and on the right can easily be located. As for the event on the left bottom, even through the source energy is relatively weak, we can still locate that passive seismic event. This is because it is not only far from the receivers, but also far from the initial source location. These two factors result in the poor source energy concentration. If we use the source image from the zero-scattered FWI in the passive seismic events monitoring, the estimate of the source position has a large error.

\section{DISCUSSIONS}

We apply multi-scattered waveform inversion to passive seismic data to invert for the velocity model and source image, simultaneously. Our numerical tests on the modified Marmousi model show that when low-frequency components in the data are missing, the zero-scattered FWI fails to reconstruct the main structure in the velocity model which leads to an inaccurate passive source location determination. Under the same conditions, the single- and 
double-scattered FWI can build a reasonably good velocity model and have an accurate passive seismic event estimation result. With higher order of scattered energy included, the convergence of the optimization is faster. The synthetic test also shows that multi-scattered waveform inversion can perform well even in noisy data.

One of the limitations of this approach is the necessity to handle events corresponding to one source at a time. This limitation is not related to the common crosstalk, but more so to locating the source and penalizing the focusing. So in the case of multi passive seismic sources, we handle them similar to the case of active seismic sources. If the recorded events overlap, we need to solve an optimization problem to deblend and reconstruct data ( $\mathrm{Li}$ et al., 2013). This limitation is common as with any inversion, we face the issue of cross talk when events from multi sources overlap (Choi and Alkhalifah, 2012).

\section{CONCLUSIONS}

We propose to use multi-scattered FWI to invert the source image and velocity model simultaneously in the frequency domain. We incorporate the spatial and wavelet components into the source function to mitigate the uncertainties of the source wavelet and source origin time of passive seismic events. Using a new objective function, we optimize the velocity model, including the multi-scattered energy, with very limited computational cost. A penalty term on source image and a regularization term for the velocity are added to the objective function to mitigate leakage. With better velocity model inversion results, the source energy is better focused at its true location in the inverted source image. Application of our approach to the Marmousi model starting from a reasonable frequency data $(3 \mathrm{~Hz}$ for single source case and $5 \mathrm{~Hz}$ for the multiple sources case) yields velocity models and source images superior to those obtained from the zero-scattered FWI.

\section{ACKNOWLEDGMENTS}

We thank KAUST for its support and the SWAG group for the collaborative environment. This work utilized the resources of the Supercomputing Laboratory at King Abdullah Uni- 
1

2

3

4

5

6

ox

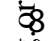

of

i 0

1

$\underset{5}{7} 2$

114

D15

16

व17

氜 8

ज9 9

ఖू०

21
.21
.22

23

25

26

38

28
30
30
30

范1

.34

跑6

$\sim 38$

3
3
-4
-4

m.4

$-42$

43

44

45

46

$-47$

- 48

ofy

\%0

5

52

53

54

55

56

57

58

59

60 versity of Science and Technology (KAUST) in Thuwal, Saudi Arabia, and we are grateful for that. 


\section{REFERENCES}

Alkhalifah, T., 2017, Optimizing the coefficients of the leading terms of the Born Series: FWI+ MVA+ more: 79th EAGE Conference and Exhibition 2017-Workshops.

Alkhalifah, T., and Z. Wu, 2014, FWI and MVA the natural way: 74th Annual International Conference and Exhibition, EAGE, Extend Abstracts.

— 2016 a, Multiscattering inversion for low-model wavenumbers: Geophysics, 81, no. 6, R417-R428.

— $2016 \mathrm{~b}$, The natural combination of full and imaged-based waveform inversion: Geophysical Prospecting, 64, 19-30.

Artman, B., I. Podladtchikov, and B. Witten, 2010, Source location using time-reverse imaging: Geophysical Prospecting, 58, 861-873.

Bozdag, E., J. Trampert, and J. Tromp, 2011, Misfit functions for full waveform inversion based on instantaneous phase and envelope measurements: Geophysical Journal International, 185, 845-870.

Choi, Y., and T. Alkhalifah, 2011, Source-independent time-domain waveform inversion using convolved wavefields: Application to the encoded multisource waveform inversion: Geophysics, 76, no. 5, R125-R134.

— 2012, Application of multi-source waveform inversion to marine streamer data using the global correlation norm: Geophysical Prospecting, 60, 748-758.

Diekmann, L., B. Schwarz, A. Bauer, and D. Gajewski, 2018, Source localisation and joint velocity model building: 80th EAGE Conference and Exhibition.

Eisner, L., P. M. Duncan, W. M. Heigl, and W. R. Keller, 2009, Uncertainties in passive seismic monitoring: The Leading Edge, 28, 648-655.

Fernandez-Berdaguer, E., 1998, Parameter estimation in acoustic media using the adjoint method: SIAM Journal on Control and Optimization, 36, 1315-1330.

Gajewski, D., and E. Tessmer, 2005, Reverse modelling for seismic event characterization: Geophysical Journal International, 163, 276-284.

Huang, G., and W. Symes, 2016, Matched source waveform inversion: space-time extension: 86th Annual International Meeting, SEG, Expanded Abstracts, 1426-1431. 
Kaderli, J., W. D. McChesney, and S. E. Minkoff, 2015, Microseismic event estimation in noisy data via full waveform inversion: 85th Annual International Meeting, SEG, Expanded Abstracts, 1159-1164.

Kamei, R., and D. Lumley, 2014, Passive seismic imaging and velocity inversion using full wavefield methods: 84th Annual International Meeting, SEG, Expanded Abstracts, $2273-2277$.

Li, C., C. C. Mosher, L. C. Morley, Y. Ji, and J. D. Brewer, 2013, Joint source deblending and reconstruction for seismic data: SEG Technical Program Expanded Abstracts, 82-87.

Plessix, R. E., 2006, A review of the adjoint-state method for computing the gradient of a functional with geophysical applications: Geophysical Journal International, 167, 495503.

Rentsch, S., S. Buske, S. Luth, and S. Shapiro, 2004, Location of seismicity using gaussian beam type migration: 74th Annual International Meeting, SEG, Expanded Abstracts, $354-357$.

Rocha, D., P. Sava, J. Shragge, and B. Witten, 2018, 3D microseismic wavefield imaging using the energy imaging condition: 80th EAGE Conference and Exhibition.

Sava, P., 2011, Micro-earthquake monitoring with sparsely sampled data: Journal of Petroleum Exploration and Production Technology, 1, 43-49.

Song, C., T. Alkhalifah, and Z. Wu, 2019, Micro-seismic event estimation and velocity analysis based on a source-focusing function: in press with Geophysics.

Song, C., T. Alkhalifah, Z. Wu, and B. Sun, 2017, Non-stationary filter used in microseismic source imaging: 87th Annual International Meeting, SEG, Expanded Abstracts, $2898-2902$.

Sun, J., Z. Xue, S. Formel, T. Y. Zhu, and N. Nakata, 2016, Full waveform inversion of passive seismic data for sources and velocities: 86th Annual International Meeting, SEG, Expanded Abstracts, 1405-1410.

Tarantola, A., 1984, Inversion of seismic reflection data in the acoustic approximation: Geophysics, 49, 1259-1266.

Virieux, J., and S. Operto, 2009, An overview of full-waveform inversion in exploration geophysics: Geophysics, 74, no. 6, WCC1-WCC26. 
Vogel, C. R., 2002, Computational methods for inverse problems: SIAM, 23.

Waldhauser, F., and W. Ellsworth, 2000, A double-difference earthquake location algorithm: Method and application to the northern hayward fault, california.: Bulletin of the Seismological Society of America, 1353-1368.

Wang, H. C., and T. Alkhalifah, 2016, Microseismic imaging using a source-independent full-waveform inversion method: 86th Annual International Meeting, SEG, Expanded Abstracts, 2596-2600.

Warpinski, N., 2009, Microseismic monitoring: Inside and out: Society of Petroleum Engineers, 61, 80-85.

Witten, B., and J. Shragge, 2017, Image-domain velocity inversion and event location for microseismic monitoring: Geophysics, 82, no. 5, KS71-KS83.

Wu, Z., and T. Alkhalifah, 2015, Simultaneous inversion of the background velocity and the perturbation in full-waveform inversion: Geophysics, 80, no. 5, 317-329.

— , 2017a, Efficient scattering-angle enrichment for a nonlinear inversion of thebackground and perturbations components of a velocity model: Geophysical Journal International, 210, 1981-1992.

— $2017 \mathrm{~b}, \mathrm{~A}$ new wave equation based source location method with full-waveform inversion: 79th EAGE Conference and Exhibition.

Xu, S., D. Wang, F. Chen, Y. Zhang, and G. Lambare, 2012, Full waveform inversion for reflected seismic data: 74th EAGE Conference and Exhibition incorporating EUROPEC 2012 .

Yao, G., and D. Wu, 2017, Reflection full waveform inversion: Science China Earth Sciences, 60, 1783-1794.

Zhang, Y., and L. Duan, 2012, Predicting multiples using a reverse time demigration: SEG Technical Program Expanded Abstracts, 1-5.

Zhdanov, M. S., 2002, Geophysical inverse theory and regularization problems: Elsevier, 36.

Zhou, H., L. Amundsen, and G. Zhang, 2012, Fundamental issues in full waveform inversion: 82nd Annual International Meeting, SEG, Expanded Abstracts, 1-5. 


\section{LIST OF FIGURES}

1 A two horizontal layer model $(*$ denotes the source location and $\triangle$ denotes the receiver location).

2 An FWI kernel with (a) zero- and (b) single-scattered energy for a horizontal layer model.

3 The combined FWI kernel with single-scattered energy for a horizontal layer model.

$4 \quad$ A dipping layer model $(*$ denotes the source location and $\triangle$ denotes the receiver location).

5 An FWI kernel with (a) zero- and (b) single-scattered energy for a dipping layer model.

6 The combined FWI kernel with single-scattered energy for a dipping layer model.

$7 \quad$ A homogeneous model with a single scatter ( $*$ denotes the true source location).

8 The source distance $\left|\mathbf{x}-\mathbf{x}_{\mathbf{s}}\right|^{2}$ in the penalty term (a) without and (b) with penalizing the large values.

9 The inverted source image using (a) the zero-scattered FWI and (b) single-scattered FWI (* denotes the source location).

10 The model image of one anomaly model.

11 Part of Marmousi model.

12 (a) The initial velocity model, (b) zero-scattered, (c) single-scattered FWI, and (d) double-scattered inverted velocity model.

13 The source image inversion results from (a) initial velocity model, (b) zero-scattered FWI inverted velocity model, (c) single-scattered FWI inverted velocity model, and (d) double-scattered inverted velocity model $(*$ denotes the initial source location and $\circ$ denotes the true source location).

14 The inverted velocity (a), the inverted source image (b) using zero-scattered FWI, the inverted velocity (c), the inverted source image (d) using double-scattered FWI with noisy data.

15 (a) The initial velocity model, (b) zero-scattered FWI inverted velocity model, (c) single-scattered FWI, and (d) double-scattered inverted velocity model. 
16 Source image inversion results from (a) initial velocity model, (b) zero-scattered FWI inverted velocity model, (c) single-scattered FWI inverted velocity model, and (d) double-scattered inverted velocity model $(*$ denotes the initial source location and o denote the true source locations). 


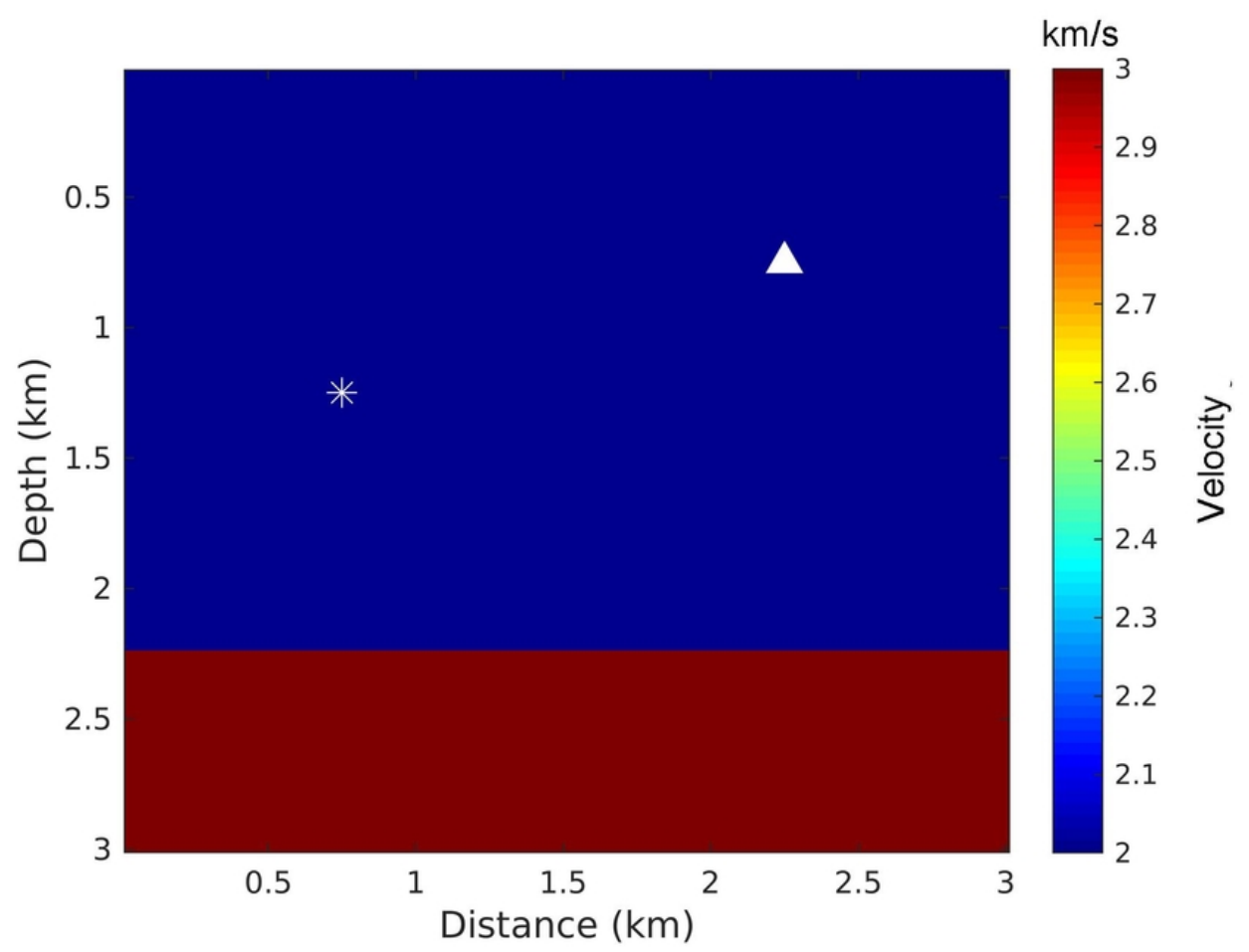

$1 \mathrm{~A}$ two horizontal layer model ( $\$ * \$$ denotes the source location and $\$ \backslash$ bigtriangleup $\$$ denotes the receiver location).

$74 \times 55 \mathrm{~mm}(300 \times 300 \mathrm{DPI})$ 
(a)

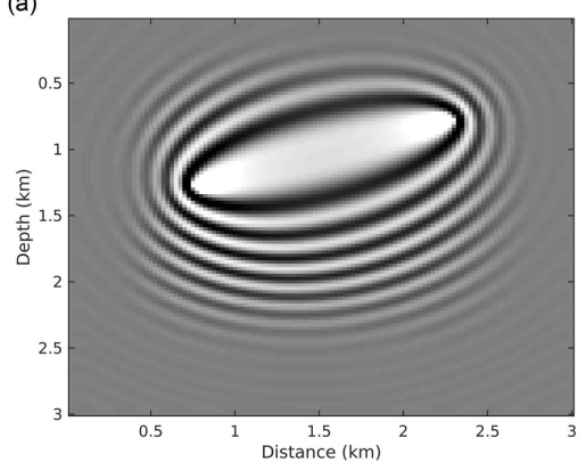

(b)

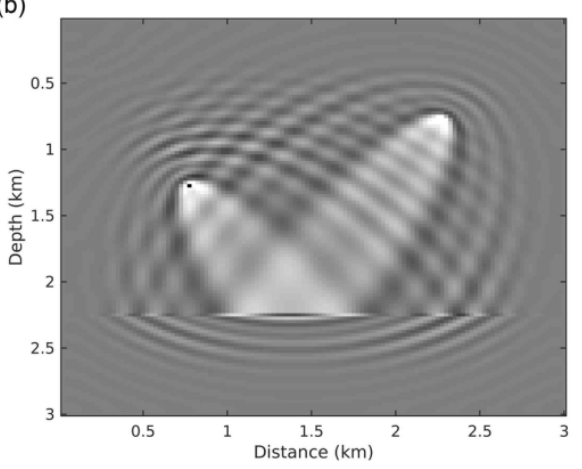

2 An FWI kernel with (a) zero- and (b) single-scattered energy for a horizontal layer model. $149 \times 56 \mathrm{~mm}(300 \times 300$ DPI $)$ 


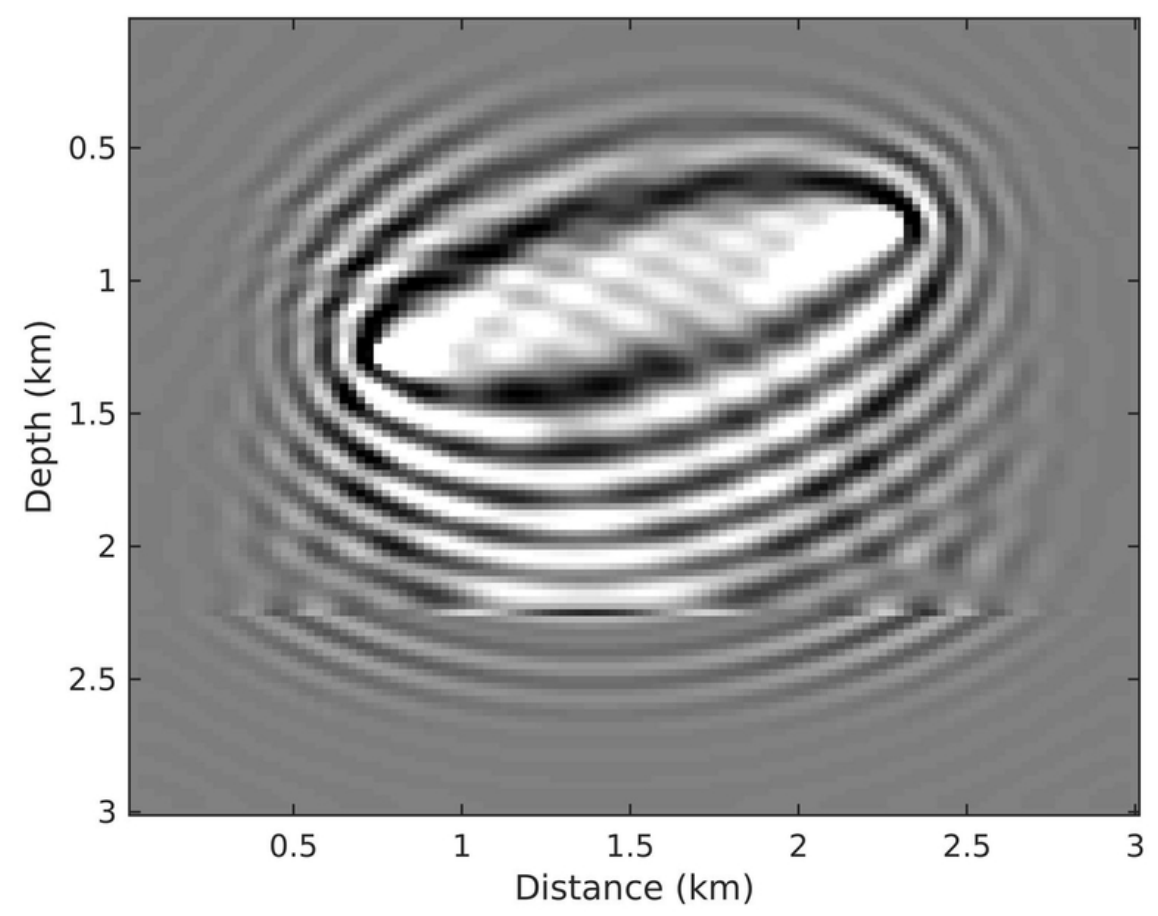

3 The combined FWI kernel with single-scattered energy for a horizontal layer model. $74 \times 56 \mathrm{~mm}$ (300 x 300 DPI) 


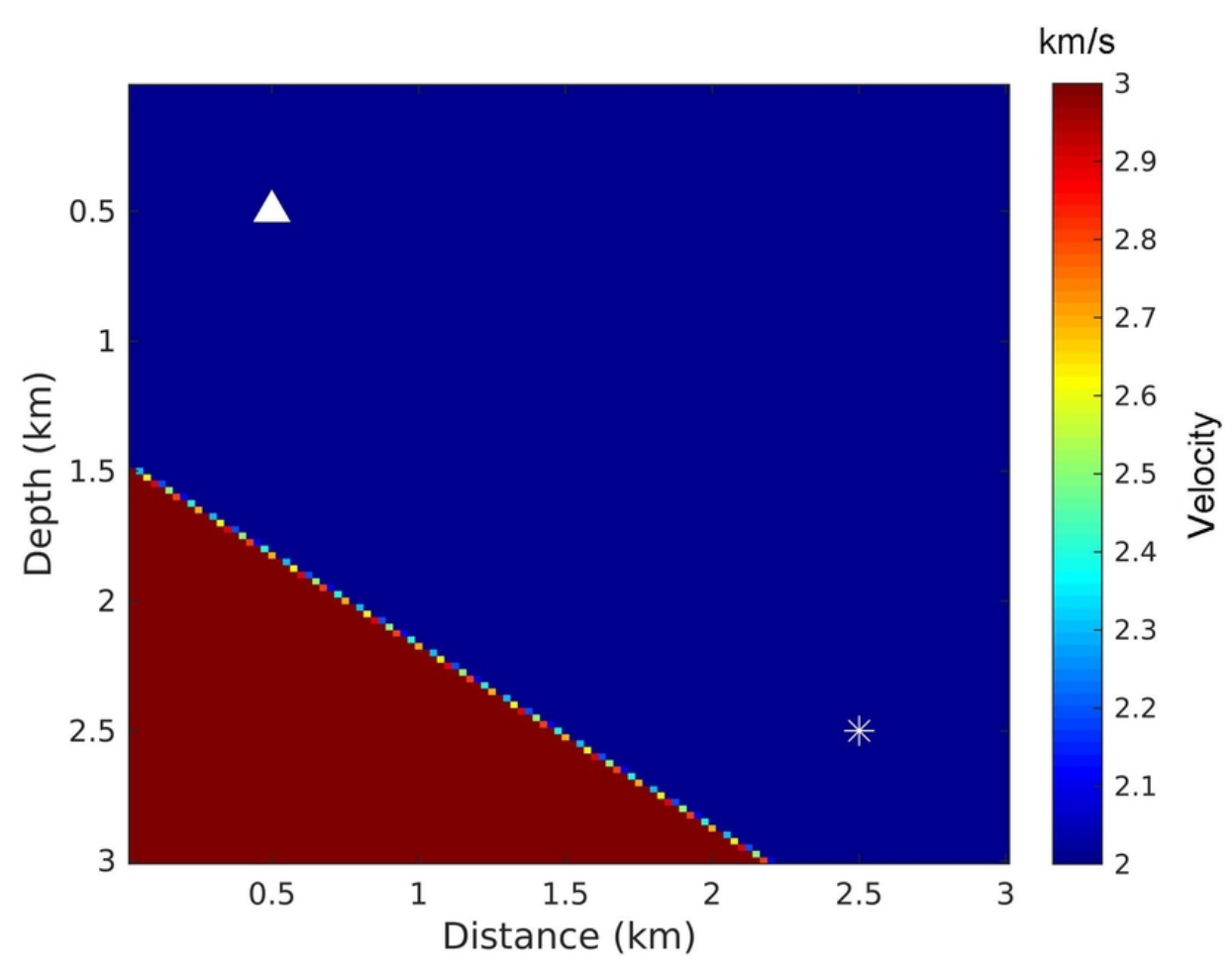

4 A dipping layer model ( $\$ * \$$ denotes the source location and $\$ \backslash$ bigtriangleup $\$$ denotes the receiver location).

$74 \times 55 \mathrm{~mm}(300 \times 300$ DPI $)$ 
(a)

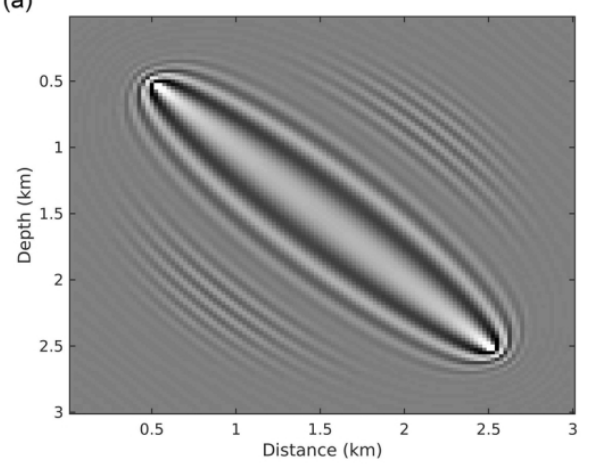

(b)

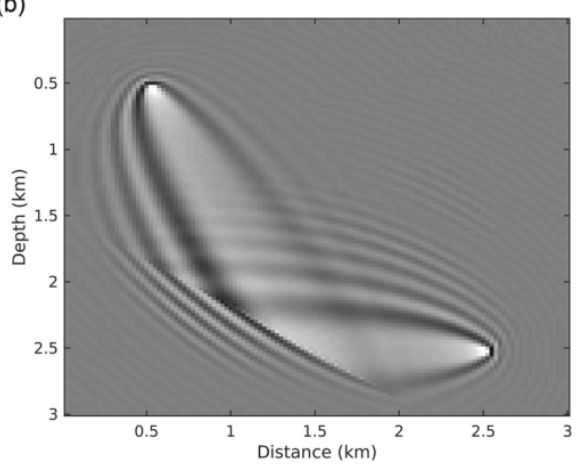

5 An FWI kernel with (a) zero- and (b) single-scattered energy for a dipping layer model. $149 \times 56 \mathrm{~mm}(300 \times 300$ DPI $)$ 


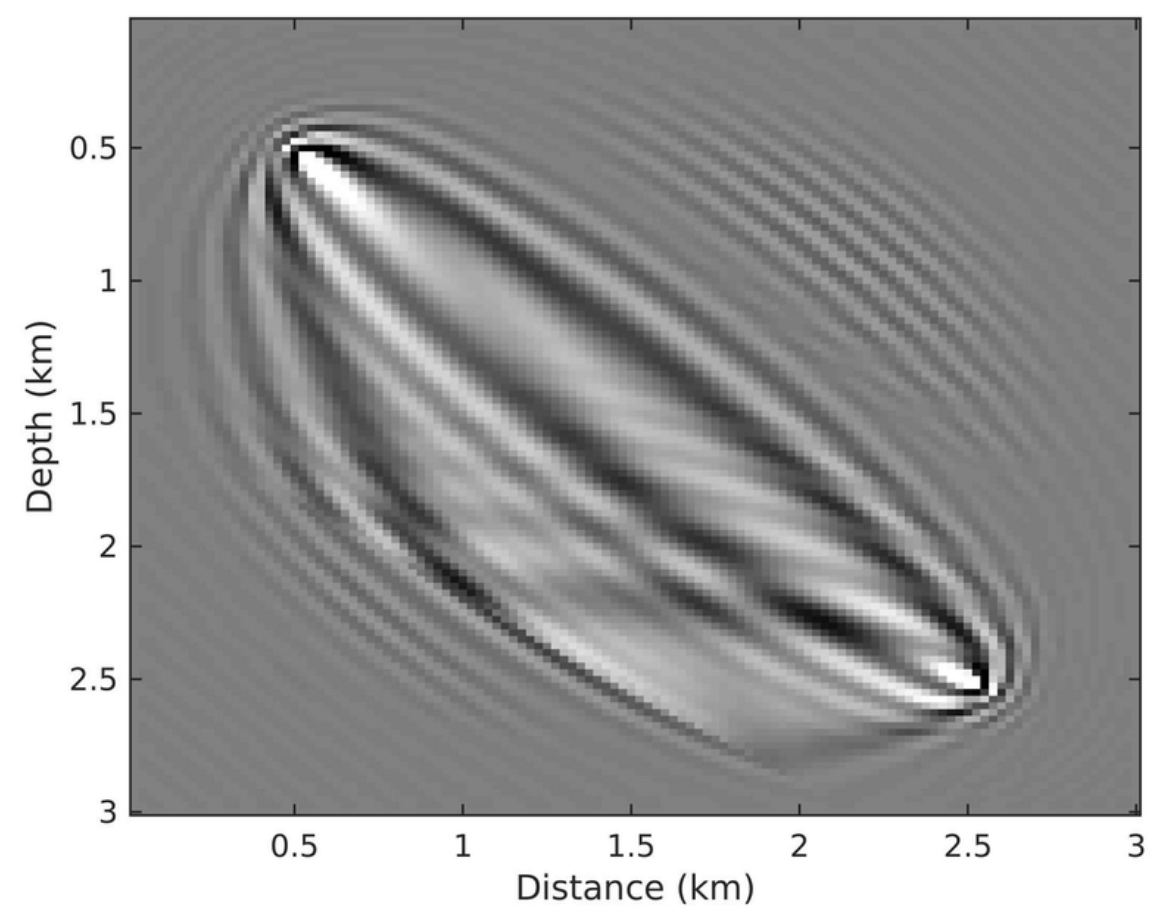

6 The combined FWI kernel with single-scattered energy for a dipping layer model.

$74 \times 56 \mathrm{~mm}$ (300 x 300 DPI)

This paper presented here as accepted for publication in Geophysics prior to copyediting and composition. (C) 2019 Society of Exploration Geophysicists. 


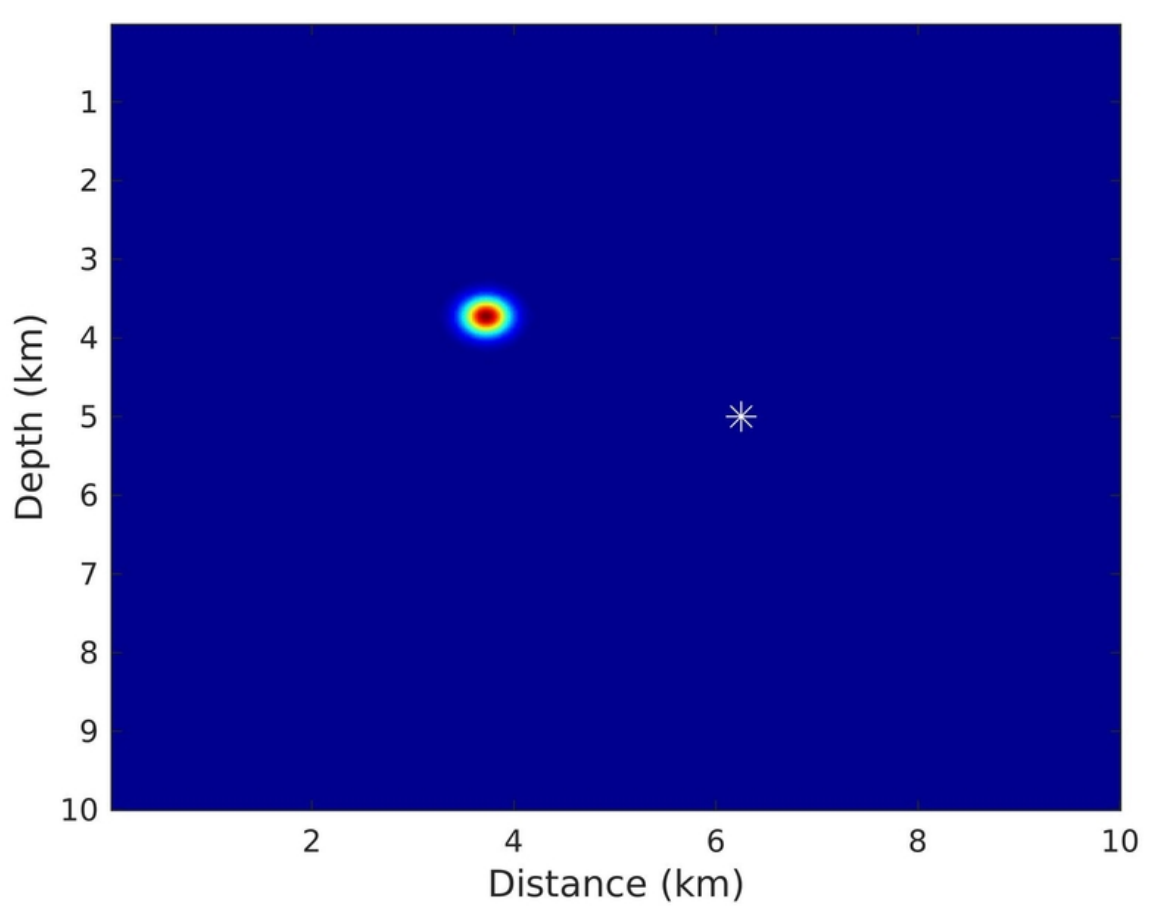

$7 \mathrm{~A}$ homogeneous model with a single scatter ( $\$ * \$$ denotes the true source location).

$74 \times 55 \mathrm{~mm}(300 \times 300 \mathrm{DPI})$ 
(a)

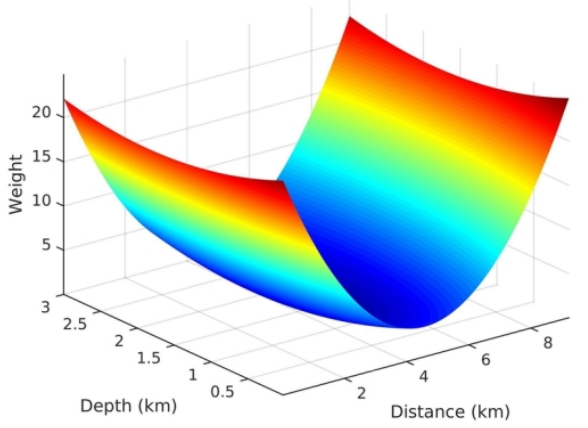

(b)

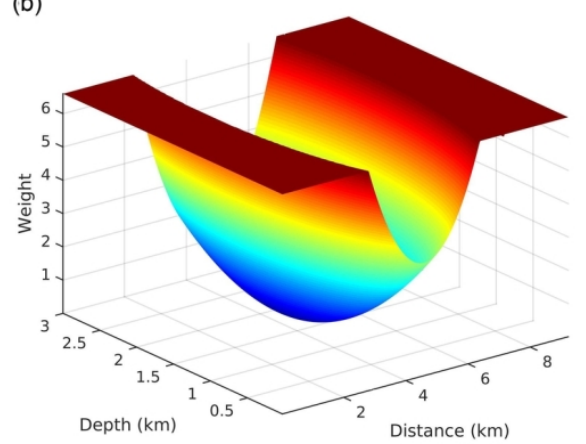

8 The source distance $\$ \backslash$ left $\mid \backslash \operatorname{mathbf}\{x\}-\backslash \operatorname{mathbf}\left\{x \_\{s\}\right\} \backslash$ right $\mid \wedge\{2\} \$$ in the penalty term (a) without and (b) with penalizing the large values.

$149 \times 56 \mathrm{~mm}(300 \times 300$ DPI $)$ 
3

4

5

6

of

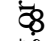

की

i 0

1

견 2

i 3

吾14

16

व17 7

ตै 8

ज9

20

21

41 (a)

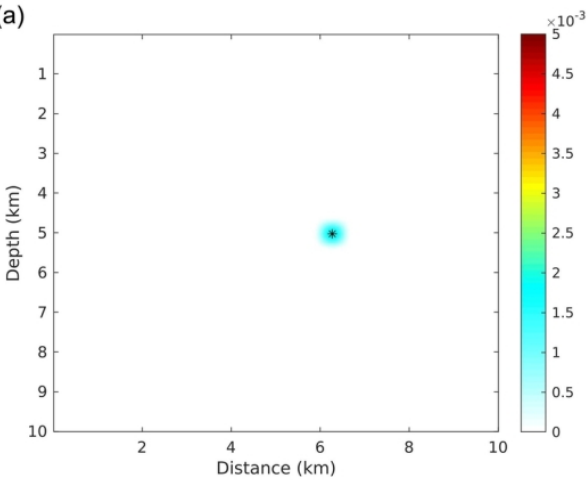

(b)

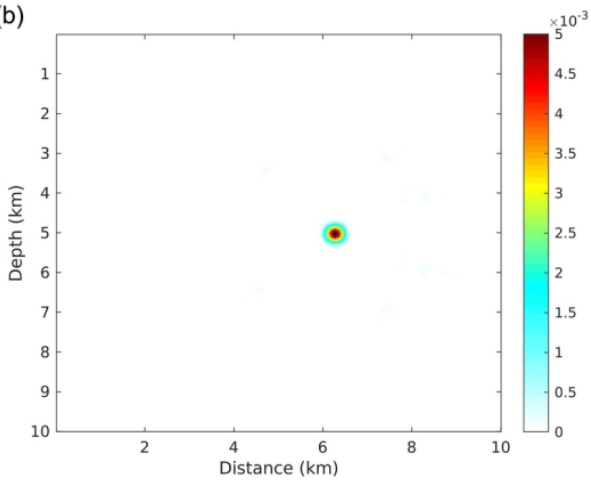

9 The inverted source image using (a) the zero-scattered FWI and (b) single-scattered FWI ( $\$ * \$$ denotes the source location).

$149 \times 56 \mathrm{~mm}(300 \times 300 \mathrm{DPI})$

This paper presented here as accepted for publication in Geophysics prior to copyediting and composition.

(C) 2019 Society of Exploration Geophysicists. 


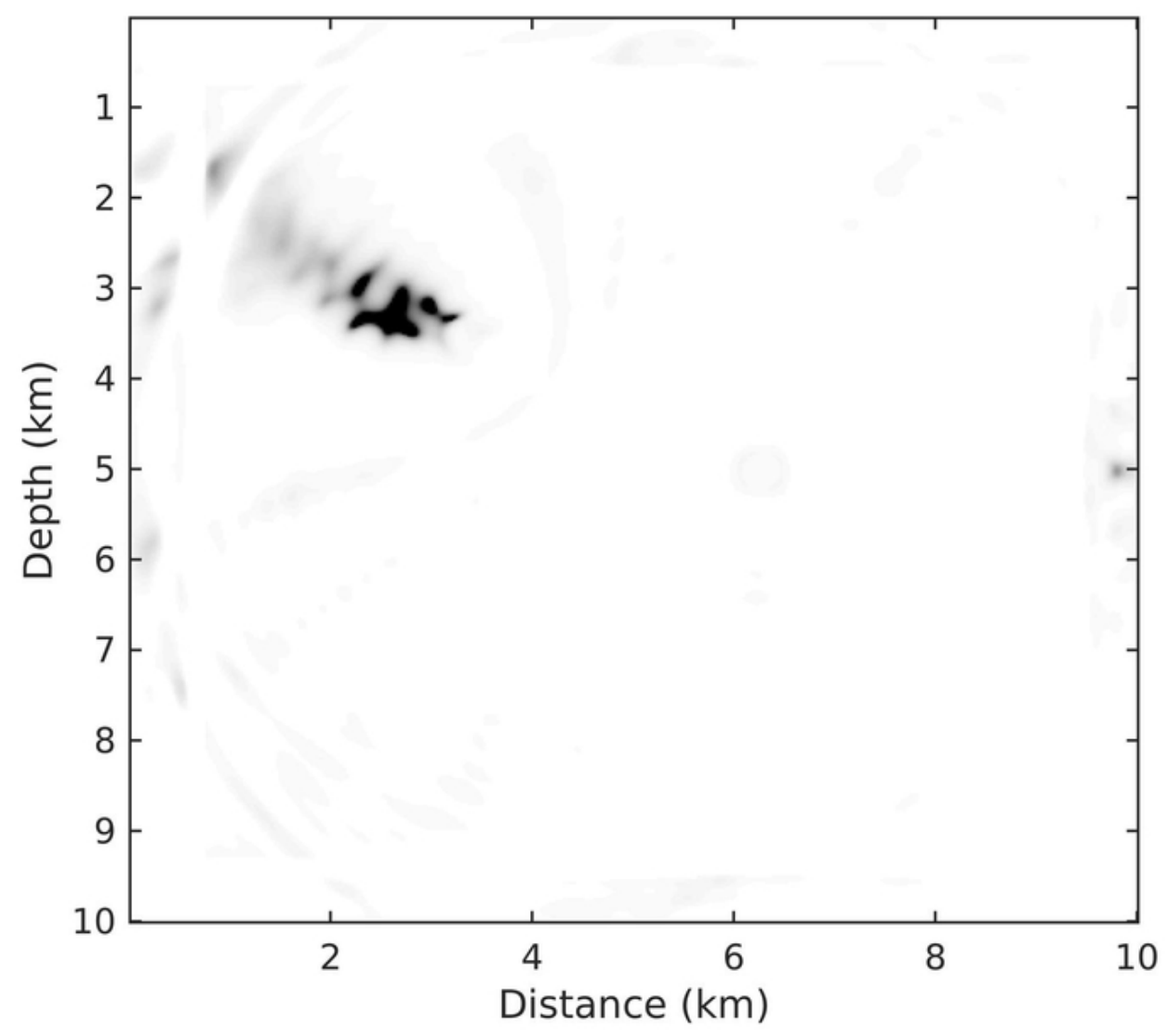

10 The model image of one anomaly model.

$60 \times 55 \mathrm{~mm}(300 \times 300$ DPI)

This paper presented here as accepted for publication in Geophysics prior to copyediting and composition.

(C) 2019 Society of Exploration Geophysicists. 


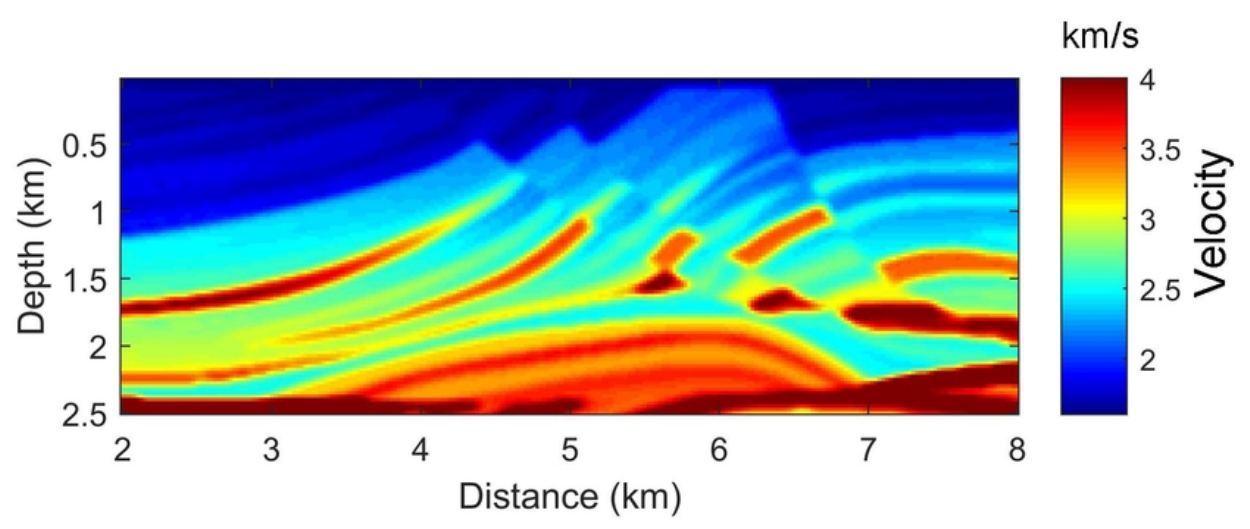

11 Part of Marmousi model.

$75 \times 30 \mathrm{~mm}(300 \times 300$ DPI $)$

This paper presented here as accepted for publication in Geophysics prior to copyediting and composition. (C) 2019 Society of Exploration Geophysicists. 
(a)

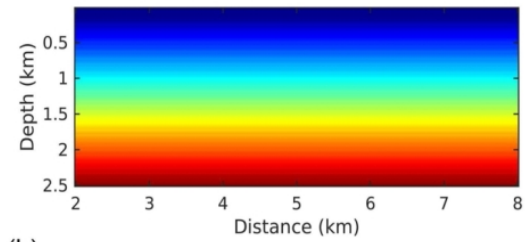

(b)

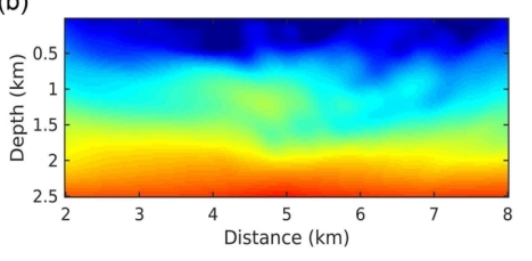

(c)

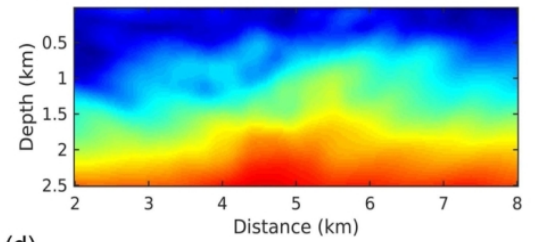

(d)

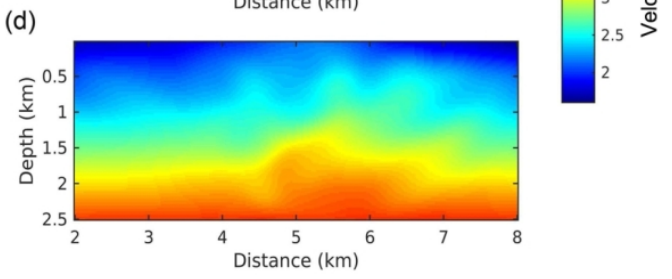

12 (a) The initial velocity model, (b) zero-scattered, (c) single-scattered FWI, and (d) double-scattered inverted velocity model.

$149 \times 62 \mathrm{~mm}(300 \times 300 \mathrm{DPI})$ 
(a)
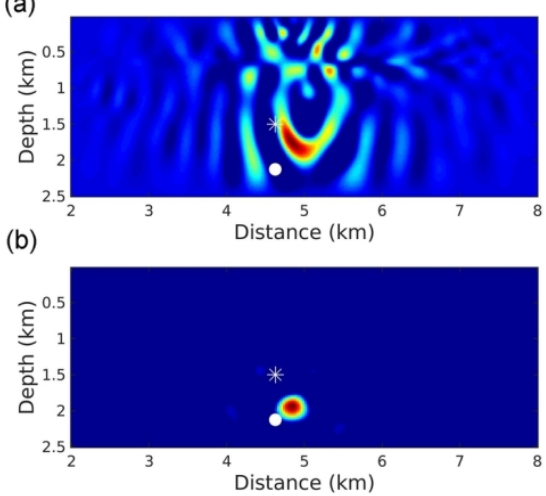

(c)

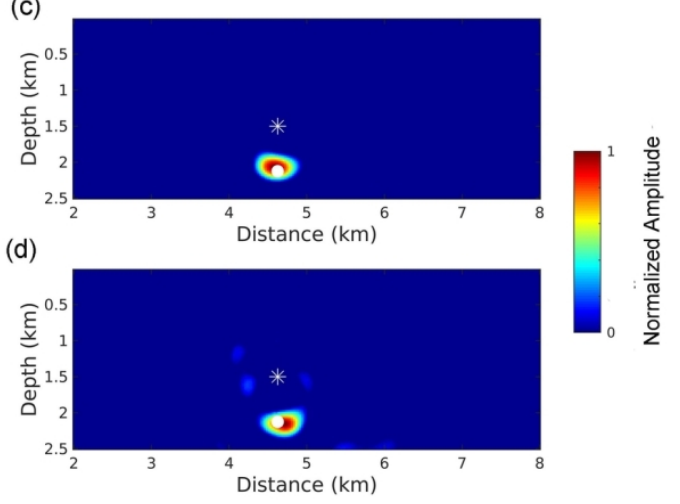

13 The source image inversion results from (a) initial velocity model, (b) zero-scattered FWI inverted velocity model, (c) single-scattered FWI inverted velocity model, and (d) double-scattered inverted velocity model ( $\$ * \$$ denotes the initial source location and $\$ \backslash$ circ $\$$ denotes the true source location).

$149 \times 59 \mathrm{~mm}(300 \times 300 \mathrm{DPI})$ 

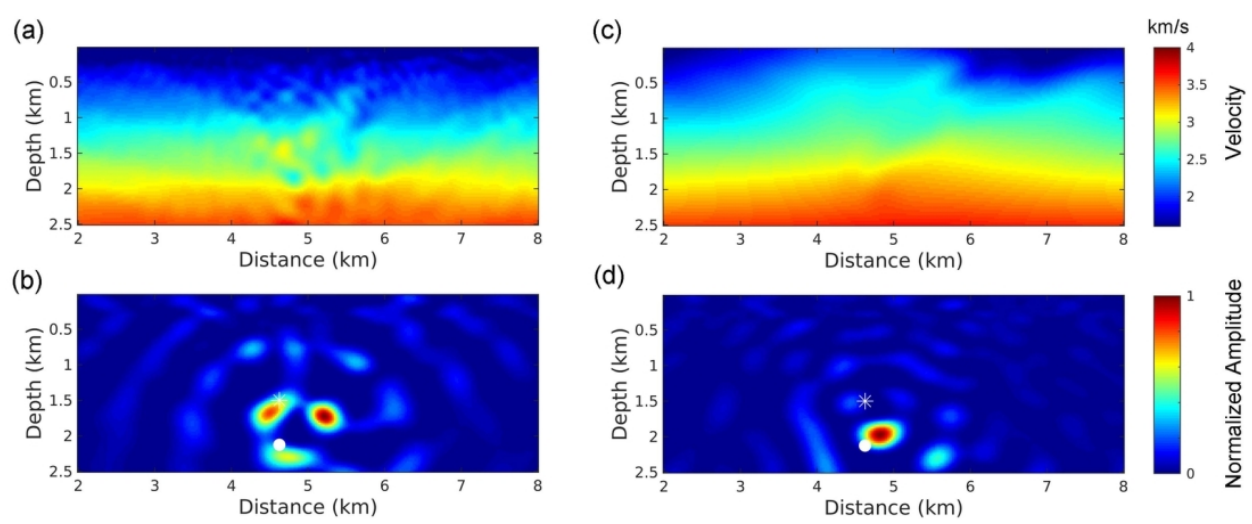

14 The inverted velocity (a), the inverted source image (b) using zero-scattered FWI, the inverted velocity (c), the inverted source image (d) using double-scattered FWI with noisy data.

$$
149 \times 59 \mathrm{~mm}(300 \times 300 \mathrm{DPI})
$$


(a)

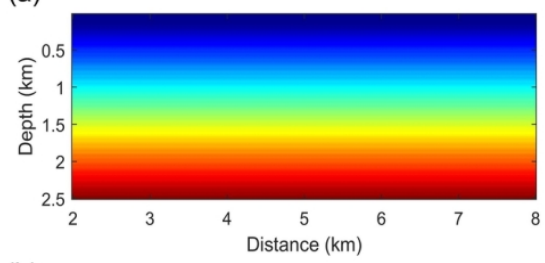

(b)

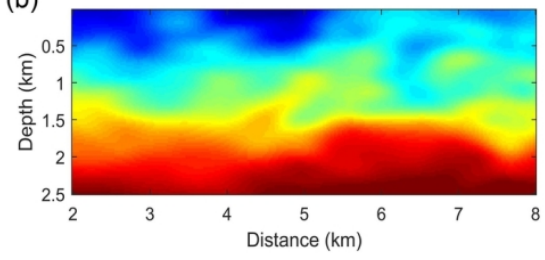

(c)

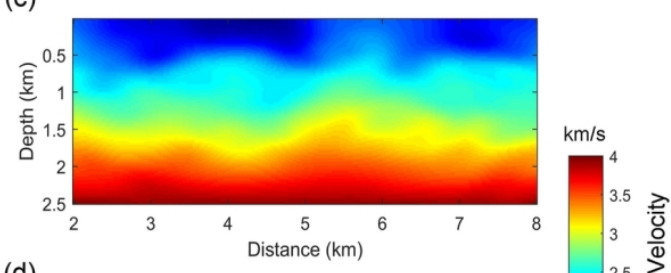

(d)

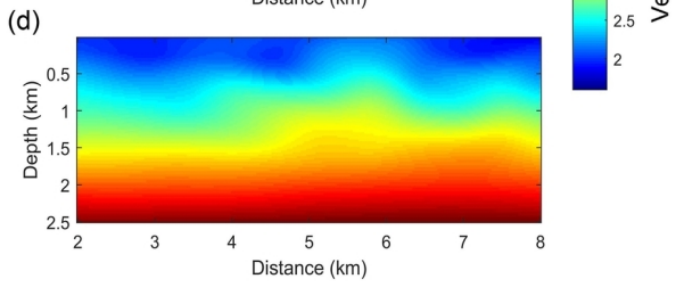

15 (a) The initial velocity model, (b) zero-scattered FWI inverted velocity model, (c) single-scattered FWI, and (d) double-scattered inverted velocity model.

$$
149 \times 64 \mathrm{~mm}(300 \times 300 \mathrm{DPI})
$$


(a)

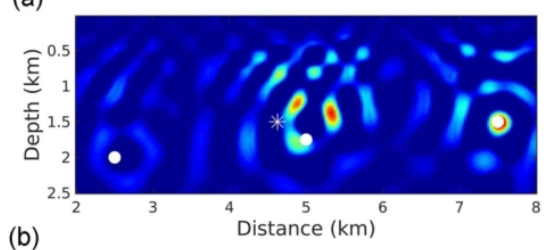

(b)

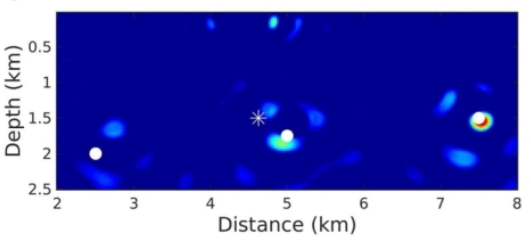

(c)

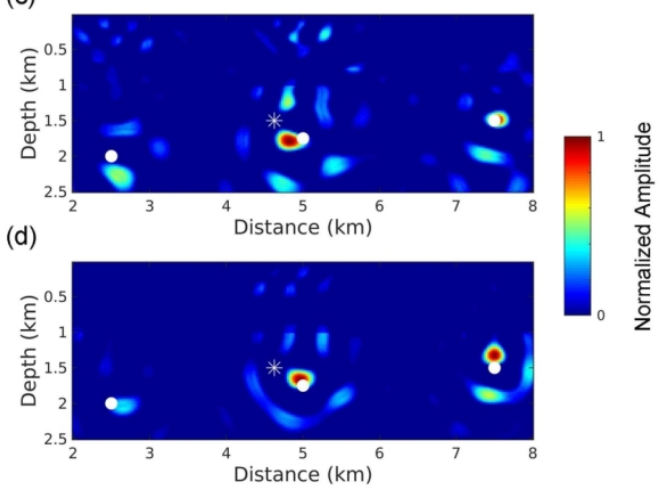

16 Source image inversion results from (a) initial velocity model, (b) zero-scattered FWI inverted velocity model, (c) single-scattered FWI inverted velocity model, and (d) double-scattered inverted velocity model ( $\$ * \$$ denotes the initial source location and $\$ \backslash$ circ $\$$ denote the true source locations).

$149 \times 59 \mathrm{~mm}(300 \times 300 \mathrm{DPI})$ 
DATA AND MATERIALS AVAILABILITY

Data associated with this research are confidential and cannot be released. 